\title{
Détermination de l'écoulement incompressible et stationnaire à travers $n$ branches en parallèle
}

\author{
R. Flatt (DME-EPFL)
}

Département de Mécanique

Ecole polytechnique fédérale de Lausanne, Suisse

\begin{abstract}
Dans une installation hydro-électrique (ou de pompage) on a en règle générale plusieurs turbines (ou pompes) connectées ensemble par un système de conduites, composé d' "embranchements " (c'est-à-dire de "branches" en parallèle) et de conduites communes. L'étude décrit un algorithme à très bonne convergence, permettant de déterminer le régime stationnaire à travers un tel embranchement, dont les branches comportent outre les conduites, des turbines, pompes, vannes, etc., réglées pour des débits quelconques.
\end{abstract}

\section{Introduction}

Du point de vue topologique un réseau de conduites hydrauliques peut être conçu comme un maillage. C'est par exemple le cas d'un réseau de distribution d'eau dans une agglomération. Lorsque l'écoulement considéré est stationnaire, les lois de la mécanique des fluides conduisent en général à deux groupes de relations :

a) les équations de continuité, au niveau des "nœuds" des "mailles" (exprimant que la somme algébrique des débits "entrants " dans le nœud est nulle),

b) les relations non linéaires des pertes de charge (dans les tronçons de conduites situés entre les nœuds).

Il faut ajouter à cela une troisième famille de relations : c) les relations non linéaires de caractéristiques de machines (turbines ou pompes) et d'organes divers (vannes, etc.).

Une approche très générale pour la résolution de tels problèmes devient rapidement complexe, lorsque le nombre de nauds augmente. Du point de vue mathématique, on est amené à résoudre simultanément un grand nombre d'équations linéarisées, impliquant des matrices d'ordre très élevé, ainsi qu'une résolution numérique gourmande en temps de calcul [1]. Par ailleurs, la linéarisation, notamment des caractéristiques de machines, implique des approximations pouvant avoir des répercussions inacceptables sur le résultat du calcul.

Dans les installations hydro-électriques la configuration topologique est particulière, du fait de l'existence d'un sens général de l'écoulement (de l'amont vers l'aval, s'il

\section{Determination of incompressible and stationary flow through $\mathbf{N}$ branches in parallel}

In a hydro-electric (or pumping) installation, as a general rule, there are several turbines (or pumps) connected together by a system of conveyances, made up of "branchings " (i.e. " branches " in parallel) and conveyances in common. The study describes an algorithm with a very good convergence, enabling the stationary regime in such an branching to be determined, the branches of which contain apart from the conveyances, turbines, pumps, valves, etc. adjusted for certain flow rates. 


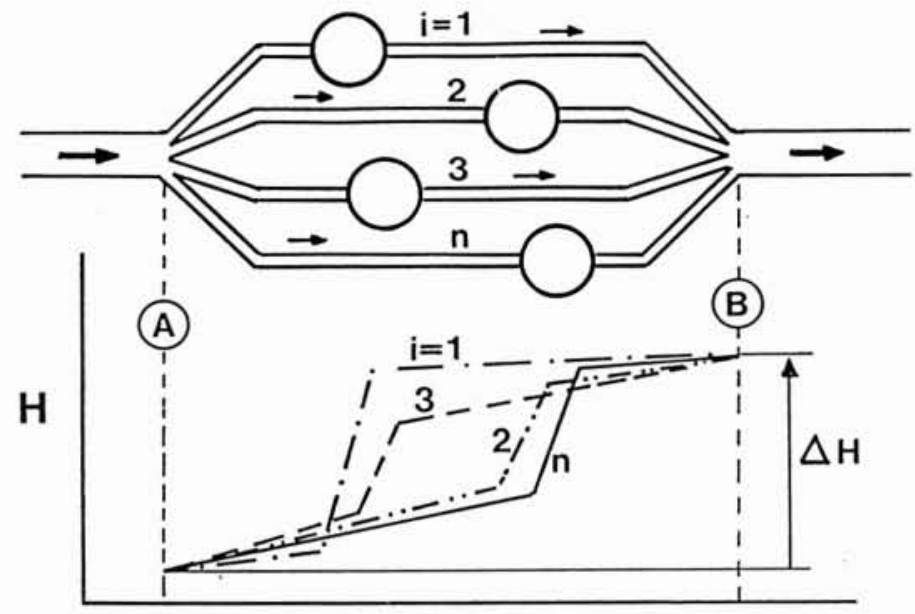

1. Représentation schématique d'un embranchement à quatre branches en parallèle. s'agit de "turbinage ", ou de l'aval vers l'amont dans le cas du " pompage "). L'ensemble du " réseau " peut en général être subdivisé en sous-réseaux du type de la figure 1. Un tel sous-réseau, que nous appellerons "embranchement", est caractérisé matériellement par un "naud aval " $A$, un "naud amont " $B$ et $n$ " branches en parallèle ", reliant chacune $A$ à $B$. Une "branche" peut comporter, outre des tronçons de conduites (de diamètres constants ou variables), des turbines, pompes, vannes, etc.

On peut tirer profit d'une telle configuration en traitant chaque " embranchement " séparément. L'écoulement qui traverse ce dernier, est caractérisé par un débit total

$$
\dot{V}_{\mathrm{tot}}=\sum_{i=1}^{n} \dot{V}_{i}
$$

où les $\dot{V}_{i}$ sont les débits-volume (a priori inconnus) dans les différentes branches. Il s'agit de déterminer :

a) les $n$ débits $\dot{V}_{i}$,

b) le $\Delta H$ commun à toutes les branches (fig. 1), où

$$
H=\frac{p-p_{\text {atm }}}{\rho g}+Z+\frac{c^{2}}{2 g}
$$

avec

$c=$ vitesse (moyenne sur la section), $g=$ gravité, $p=$ pression, $z=$ altitude et $\rho=$ densité (ou masse volumique).

Bien que l'ensemble d'un réseau peut comporter plusieurs embranchements en série (voire en parallèle), la résolution numérique de l'ensemble sera, dans certains cas, grandement simplifiée par l'approche basée sur la subdivision en embranchements. La rapidité du calcul est essentiellement due à l'absence de calcul matriciel.
Le principe de cette approche particulière étant postulé, il existe plusieurs méthodes (ou algorithmes) pour déterminer l'écoulement. Au $\$ 5$, on compare les grandes lignes d'une méthode " classique " à celle proposée dans cet article.

Une application pratique importante de la méthode présentée (qui était en fait la raison même ayant conduit à son développement !), est, paradoxalement, l'élaboration d'une méthode de calcul pour régimes instationnaires (ou plus particulièrement: transitoires), avec coups de bélier, dans les installations hydro-électriques.

La simulation sur ordinateur de régimes transitoires (engendrés par exemple par des variations de la consommation de courant électrique, entraînant la fermeture, partielle ou totale, de vannes), est un outil indispensable pour le dimensionnement de certains éléments de ces installations, tels que chambres d'équilibre ou chambres à air. Or la condition initiale d'un tel régime est en général un régime stationnaire (plus souvent appelé " régime permanent ", dans la pratique), d'où la nécessité de pouvoir déterminer ce dernier.

\section{Relations pour le problème résolu}

En hydraulique, les pertes de charge sont en général données sous la forme

$$
\left|\Delta H_{i}\right|=K_{i}+\dot{V}_{i}+. \dot{V}_{i}^{2}, \text { avec } f \ldots+=\text { fonction de } \ldots
$$

$K_{i}$ est une fonction de $\dot{V}_{i}$, qui pour une branche sans pompe ni turbine, ne dépend en général que faiblement de $\dot{V}_{i}$.

Lorsqu'il y a présence sur une " branche " d'un organe tel qu'une vanne, une turbine, une pompe, etc., le fonctionnement de celui-ci pourra également être exprimé comme fonction de $\dot{V}_{i}$ (et d'autres paramètres considérés comme fixes, ici), appelée communément "caractéristique». Nous admettrons que l'effet de cet organe sera implicitement inclu dans $\left.K_{i}+\dot{V}_{i}\right)$.

Par analogie au problème électrique bien connu de la répartition du courant dans des conducteurs montés en parallèle, nous récrivons (2) sous la forme

$$
\dot{V}_{i}=s \cdot\left|K_{i}\right|^{-1 / 2} \cdot\left|\Delta H_{i}\right|^{1 / 2} \begin{aligned}
& \text { avec } s=-1 ; 1 \text { selon la } \\
& \text { convention de signe de } \dot{V}_{i} .
\end{aligned}
$$

(La loi électrique correspondante est la loi d'Ohm, $U=R I$, récrite sous la forme $I=R^{-1} U$, où $I, R, U$ représentent le courant, la résistance et la différence de potentiel électrique.)

Une série de calculs préliminaires a montré que ce modèle simpliste présentait une convergence très lente. Pour cette raison nous élargissons le cadre de notre modèle en postulant :

$$
\dot{V}_{i}=b_{i}\left|\Delta H_{i}\right|^{1 / 2}+d_{i} \text { avec } b_{i} \text { et } d_{i}=f \dot{V}_{i} t .
$$


Des calculs ont confirmé que cette mesure a un effet très positif sur la convergence. En négligeant les pertes par mélange (respectivement: l'effet d'éjecteur) au point $B$ (fig. 1), lieu de confluence des différents débits $\dot{V}_{i}$, nous pouvons admettre que tous les $\Delta H_{i}$ sont égaux. Nous appellerons la valeur correspondante $\Delta H$. L'équation (4) devient donc :

$$
\dot{V}_{i}=b_{i}|\Delta H|^{1 / 2}+d_{i}
$$

relation qui peut être sommée membre à membre. En tenant compte de (1) ceci donne:

$$
\dot{V}_{\mathrm{tot}}=\sum_{i=1}^{n} \dot{V}_{i}=\left(\sum_{i=1}^{n} b_{i}\right) \cdot|\Delta H|^{1 / 2}+\sum_{i=1}^{n} d_{i}
$$

d'où nous tirons

$$
|\Delta H|=\left(\frac{\dot{V}_{\mathrm{tot}}-\sum_{i=1}^{n} d_{i}}{\sum_{i=1}^{n} b_{i}}\right)^{2} .
$$

L'élimination de $\Delta H$ entre (5) et (7) donne

$$
\dot{V}_{i}=\frac{b_{i}}{\sum_{i=1}^{n} b_{i}}\left(\dot{V}_{\mathrm{tot}}-\sum_{i=1}^{n} d_{i}\right)+d_{i} .
$$

Comme les $b_{i}$ et les $d_{i}$ sont des fonctions des $\dot{V}_{i}$, (8) est une formulation implicite du problème.

\section{Algorithme de résolution}

La procédure de résolution, que nous allons décrire, étant itérative, nous commençons par admettre une première distribution de $\dot{V}_{i}$, que nous notons $V_{i}^{(1)}$. Le deuxième pas consiste à évaluer branche par branche les $\Delta H_{i}^{(1)}\left(V_{i}^{(1)}\right)$. Ces calculs donneront des $\Delta H_{i}^{(1)}$ différant les uns des autres, et ne vérifiant donc pas les hypothèses nécessaires à l'élaboration de (7) et de (8) !

Ici nous faisons intervenir un élément heuristique (nous laissons l'élaboration d'un étayement plus rigoureux à un mathématicien !) : Nous déterminons les $b_{i}$ et $d_{i}$ à partir des relations approximatives obtenues en remplaçant dans (5) $\Delta H$ par les $\Delta H_{i}$ obtenus lors des deux itérations précédentes $(j-1)$ et $j$. Nous obtenons ainsi pour le pas d'itération $(j+1), n$ systèmes linéaires à deux équations et deux inconnues, de la forme :

$$
\begin{aligned}
& {\left[\left|\Delta H_{i}\right|^{(j-1)}\right]^{1 / 2} \cdot b_{i}+d_{i}=\dot{V}_{i}^{(j-1)}} \\
& {\left[\left|\Delta H_{i}\right|^{(j)}\right]^{1 / 2} \cdot b_{i}+d_{i}=\dot{V}_{i}^{(j)}}
\end{aligned}
$$

dont les solutions sont immédiates :

$$
b_{i}=\frac{\dot{V}_{i}^{(j)}-\dot{V}_{i}^{(j-1)}}{\left[\left|\Delta H_{i}\right|^{(j)}\right]^{1 / 2}-\left[\left|\Delta H_{i}\right|^{(j-1)}\right]^{1 / 2}}
$$

et

$$
d_{i}=\dot{V}_{i}^{(j)}-\left[\left|\Delta H_{i}\right|^{(j)}\right]^{1 / 2} \cdot b_{i}
$$

(Les équations (9) et (10) sont à appliquer séparément aux $n$ branches, $i=1,2, \ldots, n)$.

Comme quatrième et dernier pas, il reste à évaluer $\sum_{i=1}^{n} b_{i}$ et $\sum_{i=1}^{n} d_{i}$ et à utiliser (8) pour la détermination d'une nouvelle série de $\dot{V}_{i}$ correspondant à l'itération appelée plus haut $(j+1)$.

Il convient de relever ici que ces $\dot{V}_{i}$ vérifient automatiquement la relation (1), quelles que soient les valeurs des $b_{i}$ et des $d_{i}$ ! (On le vérifie facilement en effectuant la somme sur tous les $i$ du membre de droite de (8)). C'est dans ce fait que réside un attrait majeur de la méthode, qui débouche ainsi sur un procédé itératif à un seul niveau (contrairement au procédé décrit plus loin, au §5.)

A la fin d'un pas d'itération, il convient de vérifier la convergence. Ceci peut se faire en comparant au $\left|\Delta H_{1}\right|$ tous les autres $\left|\Delta H_{i}\right|$, et en imposant une valeurlimite pour l'écart admis :

$$
\left|\frac{\left|\Delta H_{i}\right|}{\left|\Delta H_{1}\right|}-1\right|<\varepsilon \text { avec } i=2,3, \ldots, n \text {. }
$$

Lorsque ces $(n-1)$ conditions sont vérifiées, on terminera le calcul par l'évaluation pondérée de $|\Delta H|$ obtenue à partir de (7).

\section{Astuce pour les $m$ premières itérations}

Lors de la première itération, conduisant aux $\dot{V}_{i}^{(2)}$, on ne dispose pas encore de deux séries de paires de valeurs $\dot{V}_{i}$ et $\left|\Delta H_{i}\right|$, puisqu'il n'y a pas de $\dot{V}_{i}^{(0)}$, ni de $\left|\Delta H_{i}^{(0)}\right|$. Il convient alors d'utiliser l'algorithme simplifié consistant à admettre tous les $d_{i}=0$, c'est-à-dire de se baser sur le modèle relatif à la relation (3).

Notons que l'on pourrait très bien baser toutes les itérations sur ce modèle, mais (comme dejà mentionné au $\S 2$ ), les calculs effectifs montrent que la convergence est alors relativement lente (fig. 2, page suiv.). Relevons toutefois que la convergence de ce procédé est mieux assurée de manière générale, que celle décrite au $\$ 3$ (qui est basée implicitement sur une extrapolation plus risquée). $\mathrm{Au}$ cas où un problème de convergence se manifestait, on pourrait utiliser avantageusement la combination des deux modèles, en effectuant pour commencer $m$ itérations à l'aide du modèle "éq. (3) ", puis en terminant par le modèle du $\$ 3$. La valeur idoine de $m$ devrait être recherchée par tâtonnements, puisqu'elle serait tributaire de l'installation à analyser. 


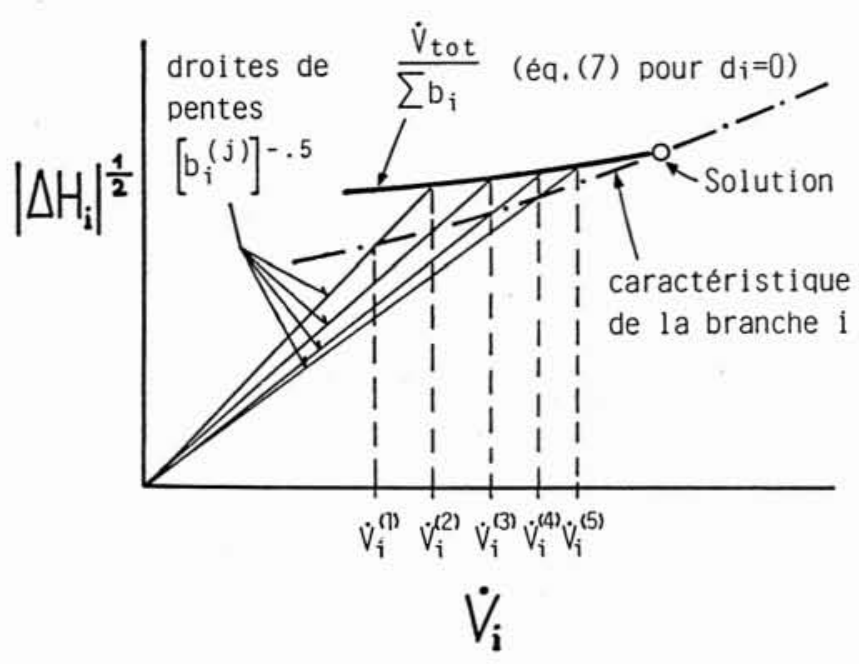

2. Illustration de la bonne, mais relativement lente convergence d'un calcul basé sur le postulat trop simpliste $\Delta H_{i}=K_{i} \cdot \dot{V}_{i}^{2}$, respectivement $\dot{V}_{i}=b_{i} \cdot\left|\Delta H_{i}\right|^{1 / 2} ;\left(d_{i}=0\right)$.

\section{Comparaison avec un procédé classique}

Au $\S 10.5$ («Pipes in parallel») de [2] les auteurs décrivent le procédé classique que nous reproduisons ici, en le modifiant très légèrement :

1) Choix d'une valeur initiale estimée $\dot{V}_{1}$.

2) Calcul de $\left.\Delta H_{1}=t \dot{V}_{1}\right)$.

3) Détermination de $\dot{V}_{2}, \dot{V}_{3}, \ldots, \dot{V}_{n}$, tels que les $\Delta H_{i}$ correspondants soient tous égaux à $\Delta H_{1}(1 \pm \varepsilon)$.

4) $\dot{V}_{\text {tot }}^{*}=\dot{V}_{1}+\dot{V}_{2}+\cdots+\dot{V}_{n}$.

5) Nouvelle valeur $\dot{V}_{1 \text {, nouveau }}=\frac{\dot{V}_{\text {tot }}}{\dot{V}_{\text {tot }}^{*}} \cdot \dot{V}_{1}$.

6) Contrôle de la convergence. Si celle-ci n'est pas encore satisfaite, retour au point 1).

Ce procédé présente un désavantage notoire: en effet, les caractéristiques de turbines et de pompes sont communément formulées comme fonctions de $\dot{V}_{i}$ (ou d'une forme adimensionnelle de cette grandeur). Ceci convient parfaitement pour le calcul de $\left.\Delta H_{1}=f \dot{V}_{1}\right)$, mais conduit par contre en général (au point 3)) à une détermination itérative des $\dot{V}_{2}, \dot{V}_{3}, \ldots, \dot{V}_{n}$. En effet chaque branche étant constituée d'un ensemble de $q$ éléments disposés en série (par exemple: conduite + vanne + turbine + conduite), on obtient

$$
\Delta H_{i}=\sum_{i=1}^{q} \Delta H_{i, I}+\dot{V}_{i}+
$$

qui est une relation implicite en $\dot{V}_{i}$. Il en résulte pour l'ensemble du calcul, une procédure itérative à deux niveaux imbriqués.
Remarque: Le pas de calcul 3) montre que plus le nombre de branches $n$ est élevé, plus il y a d'itérations supplémentaires, comparé au procédé exposé aux $\$ 3$ et 4. Comme $n$ varie d'une installation à l'autre, et que le nombre d'itérations nécessaires pour déterminer chacun des $\dot{V}_{2}, \dot{V}_{3}, \ldots, \dot{V}_{n}$, dépend fortement de la caractéristique de la branche correspondante $\Delta H_{i}\left(\dot{V}_{i}\right)$, de l'algorithme utilisé, ainsi que de la précision imposée (arbitrairement), il n'existe pas de "calcul-type ", permettant une comparaison générale entre les deux procédés. Pour cette raison nous renonçons à présenter une application particulière, qui courrait le risque d'être interprêtée comme tendancieuse.

\section{Conclusions}

1) Bien que les problèmes d'écoulements hydrauliques présentent en général un comportement non linéaire $\left(\Delta H=K+\dot{V} \nsucc \cdot \dot{V}^{2}\right)$, une analogie électrique linéaire (loi d'Ohm et éq. (3)) a fourni l'idée de base pour un algorithme à très bonne convergence, permettant de déterminer la répartition des débits $\dot{V}_{i}$, dans un embranchement de conduites en parallèle, comportant des turbines, pompes, vannes, etc.

2) L'algorithme présenté n'est pas restreint à l'application hydraulique décrite ici. Il peut être utilisé plus généralement pour des problèmes analogues (branches en parallèle), caractérisés par la conservation d'un flux (tel que le débit-masse d'un écoulement compressible stationnaire, ou le courant électrique).

3) La procédure indiquée comporte un élément heuristique. L'auteur n'a pas connaissance d'une démonstration rigoureuse, et serait heureux de susciter l'intérêt d'un mathématicien pour cet aspect du problème. Il faut cependant être conscient du fait qu'une telle analyse risque fort d'être tributaire des caractéristiques de l'installation, et de ce fait, ne pourra guère être de portée générale !

Remerciements : L'auteur remercie M. J.-E. Prénat, chef du groupe CODY (Comportements dynamiques) de l'IMHEF (Institut de machines hydrauliques et de mécanique des fluides) de l'EPFL (Ecole polytechnique fédérale de Lausanne) pour les discussions concernant le présent article.

\section{Bibliographie}

[1] Tschumy A. et Wavre C. - «Le calcul des régimes transitoires dans les installations de turbinage-pompage complexes et de grandes dimensions ". Charmilles, Informations techniques, $\mathrm{N}^{-14}$, p. 27/33, 1975. (Paru également comme "Communication II 4 " au Symposium 1974 de l'AIRH).

[2] Streeter V.L. and Wylie E.B. - « Fluid Mechanics, First Metric Edition ». McGraw Hill, 1983. 